Journal of Psycholinguistic Research, Vol. 21, No. 2, 1992

\title{
Cross-Linguistic Contrasts of Verification and Answering Among Children
}

\author{
M. Michael Akiyama ${ }^{1}$
}

This review article examines how children verify a statement (e.g., You are a child. Right or wrong?) and answer a corresponding question (e.g., Are you a child? Yes or no?) in English, French, Japanese, and Korean. While people verify affirmative statements and answer affirmative questions similarly across the four languages, they answer negative questions differently across the four languages. In English, answering negative questions works in a way opposite to verification (e.g., Are you not a child? Yes; You are not a child. Wrong). In French, si is used in the place of the yes response in English. In Japanese and Korean, answering negative questions works in a way similar to verification (e.g., Are you not a child? No; You are not a child. Wrong). The effects of these linguistic characteristics are examined. Findings are: (1) All children across the four languages appear to start answering negative questions using the English system; (2) English-speaking children find verifying negative statements more difficult than answering the corresponding questions but Japanese-speaking children find it less difficult; and (3) while English-speaking and Korean-speaking children find true negative statements more difficult to verify than false negative statements, Japanese-speaking children find them less difficult. Language-universal and language-specific processes in verification and answering are discussed.

The present article attempis to provide a framework within which psy-

A portion of this article was supported by NIMH grant 1 RO3 MH34606-01. The author wishes to thank Marilyn Shatz, Kazuko Inoue, and Edward Shoben for their willingness to discuss some of the theoretical issues presented in this paper, and John Macnamara, Brian MacWhinney, Sam Glucksburg, and Susan Gelman for their helpful suggestions. The author also wishes to thank Rick Pollack and Belinda Biscoe for their helpful reading of an earlier version of this manuscript.

${ }^{1}$ Address all correspondence, including requests for reprints, to M. Michael Akiyama, Department of Behavioral Sciences, University of Michigan-Dearborn, Dearborn, Michigan 48128. 
cholinguistic processes of verification and answering can be conceptualized across languages. The framework specifies which processes are universal across languages and which processes are specific to a language. The verification and answering systems are closely related to and interact with each other. The interaction is most pronounced while the systems are being acquired. General issues to be dealt with include (a) the form of representations involved in verification and answering and (b) possible factors compatible with children's performance in verification and answering tasks. These issues are discussed in light of previous cross-linguistic findings (Akiyama, 1979, 1984; Akiyama \& Guillory, 1983; Akiyama, Takei, \& Saito, 1982; Choi, 1991; Kim, 1985; Kim, Shatz, \& Akiyama, 1990) and findings on the acquisition of Japanese (Clancy, 1985; Noji, 1974, 1976; Okubo, 1967). Although the framework was developed initially based on English and Japanese data, it is now extended using the typological classification of answering systems by Pope (1973).

According to Pope (1973), the relationship between verification and answering varies considerably across languages. The variability may be best understood in relation to one important constant aspect across languages: In almost all languages, including English, French, Japanese, and Korean, answering affirmative yes-no questions is similar to verifying affirmative statements. For example, children say yes to the question Are you a child? and right to the statement You are a child. Yes answers and right or true responses are semantically positive, whereas no answers and wrong or false responses are negative. Semantically positive morphemes are used to express agreement with the content of respective sentences, whereas negative morphemes are used to express disagreement.

In answering negative questions, positive morphemes are not always used to express agreement and negative morphemes are not always used to express disagreement with the content. There is a vast variability across languages. In some languages, including Japanese, Chinese, Korean, and Navaho, negative questions are answered in terms of the agreement-disagreement dimension, i.e., similarly to the way in which negative statements are verified. For example, Are you not a baby? is answered Yes (true), I am not a baby. Are you not a child?, on the other hand, is answered $N o$ (false), I am a child. In these languages, answering negative questions is basically identical to verifying the corresponding statements. In answering negative questions, positive morphemes are used to express agreement and negative morphemes are used to express disagreement.

At the other extreme are English and Spanish, in which a negative 
question is answered as if there were no negatives in the question. For example, Are you not a baby? and Are you a baby? are answered No, I am not a baby. One can ignore a negative to answer a negative question correctly but one must process a negative to verify the corresponding statement correctly. In answering negative questions, positive and negative morphemes are used in a way consistent with the succeeding main clause.

In between these two groups, are French and German which express disagreement only when the statement form of negative questions is false negative. For example, si and doch are used in place of a yes response to the question Are you not a child? in the respective languages. These morphemes are used to express disagreement with the falsehood of the statement. Thus, negatives must be processed to answer false negative questions correctly. In answering true negative questions, negative morphemes are used to express negation in the succeeding main clause.

The first set of predictions can be made from the above linguistic analyses. With affirmative sentences, children of all languages should find it equally difficult to verify statements and answer questions. With negative sentences, children should find it equally difficult to verify statements and answer questions when the two systems converge, whereas they should find it more difficult to verify than to answer questions, or vice versa, when the two systems diverge.

The second set of predictions is based on the verification models for English (Carpenter \& Just, 1975; Clark \& Chase, 1972). These models predict that true negatives (e.g., You aren't a baby) are more difficult to verify than false negatives (e.g., You aren't a child). According to the models, children represent a statement and compare it with their own knowledge about the topic of the statement. Suppose children hear negative sentences such as You aren't a baby and understand deictic terms (e.g., you and $I$ ). They represent the statement as $I$ am not $a$ baby and then compare it with the knowledge I am a child. They find two mismatches in the process of comparison, one between I am not and I am and the other between $a$ baby and $a$ child. Upon hearing false negative sentences (e.g., You aren't a child), in contrast, children compare the statement representation $I$ am a child. They find only one mismatch between I am not and I am. Thus, true negatives should be more difficult to verify than false negatives.

The third set of predictions derives from the answering model for English (Akiyama, Brewer, \& Shoben, 1979). Because of the variability of the answering system, there is no single answering model to be tested across languages. The English answering model specifies psycholinguis- 
tic processes in the following way: Children represent the question and knowledge without negative markers, since the affirmative-negative distinction does not make a difference in response. Thus, negative questions should be processed just like the corresponding affirmative questions.

The model for English answering that proposes affirmative representations for all questions does not mean that children do not process the negative in general. Clearly, they produce and comprehend negative sentences as soon as they begin to talk (Brown, 1973; Klima and Bellugi, 1973; McNeill and McNeill, 1968). The model simply states that children need not represent the negative to answer correctly and therefore answering is less difficult than verification. The Japanese answering model, in contrast, predicts that answering and verification are equally difficult, since answering is basically identical to verification.

These predictions will be tested against cross-linguistic studies in English, French, Japanese, and Korean. Although the four language comparisons cannot adequately address the issue of universality, any difference in difficulty order across these languages should question the idea that language processes are universal across languages.

\section{METHODS}

All of the data reported in the present article were collected in a similar way with the exception of those by Choi (1991). In most cases, the subjects were generally from a middle-class background, their ages were 3 through 7, and the number of boys and girls was equal in one study (Akiyama and Guillory, 1983) and roughly balanced in the others. The materials were sampled from sentences that children hear often in everyday life. Sentences were presented with or without pictures. The examples of sentences with pictures include This is a bird, Is this a bird?, This isn't a dog, and Isn't this a dog? The examples of sentences without pictures include You are a baby, Aren't you a child?, You are not a mother, and Do you like cookies? There were four types of statements and questions: true affirmatives, false affirmatives, true negatives, and false negatives. Sentences in a given task were randomly ordered.

After establishing rapport with the subject, the experimenter gave the subject a few practice items, including negative sentences. Except in Kim (1985), the subject was given practice items involving his or her name, which turned out to be the easiest of all the negative statements used. Only those subjects who correctly responded to the two items You aren't Mary and You aren't (child's name) participated in the experiment. 
Following successful completion of these items, the verification and/or answering tasks were immediately given. Children were instructed to say right or wrong for the verification task and yes or no for the answering task in their own language. In Kim (1985), the subject was given four practice trials in which the subject was asked to judge a puppet's statements to be right or wrong.

Analyses of variance were performed on the number of errors for each type of statement and question. In some studies $F_{1}$ and $F_{2}$ refer to test statistics appropriate to the design, with subjects and items, respectively, as random effects. The notation $\min F^{\prime}$ refers to the test statistic for the design with the two effects combined (Clark, 1973). The error rates are used instead of the number of errors for easier comparison across studies.

Choi (1991) studied only answering using both the longitudinal and cross-sectional method. In the longitudinal method, two English-speaking children, four French-speaking children, and two Korean-speaking children participated in the study. In the cross-sectional study, 12 children from each language group participated. Their ages ranged from 1;9 to $3 ; 0$. In the longitudinal study, children were asked four types of questions in a naturalistic setting. For example, a child was asked $I s$ this a bird? while the investigator or a mother pointed to a bird in a picture book. In the cross-sectional study, the children were shown pictures of objects (e.g., boat, truck, and crayon) and animals (e.g., lion, monkey, and bear) and were asked eight true affirmative questions, eight false affirmative questions, eight true negative questions, and eight false negative questions.

\section{EVIDENCE}

All children across the languages showed a similar pattern in answering questions before showing a language-specific pattern.

Choi (1991) reported that all children across the three languages (i.e., English, French, and Korean) followed the same pattern in answering yes-no questions before using a pattern specific to their language. In her study, findings from the longitudinal study and crosssectional study converged. In the following, general findings are reported with the age range and the range of the mean length of utterances (MLU). At the initial stage, children (age range 1;8-1;11 and MLU range 1.21.4) used affirmative or negative responses to fulfill the interactional requirement of answering questions. At the second stage, children (age 
range 1;9-2;5 and MLU range 1.2-2.0) began to use affirmative and negative responses to affirmative questions correctly and treat negative questions as if they were affirmative questions. At the third stage, children (age range 2;2-2;6 and MLU range 1.9-3.4) used the agreementdisagreement dimension particularly for true negative questions and elaborate responses to false negative questions (e.g., the response ball to the question Isn't this a ball? was judged to be elaborate). Choi's second stage corresponds to the English system and her third stage to the Japanese and Korean system. These findings strongly suggest the universal processing in answering questions at the earliest stages of language development.

Only after the third stage did children begin to use the responses specific to their respective language. At the fourth stage (age range 2;63;3 and MLU range 2.2-4.4), English-speaking children and Frenchspeaking children used the English system and the French system, respectively, more than $80 \%$ of the time. Korean-speaking children used positive morphemes to express agreement more than $80 \%$ of the time for true negative questions and used the elaborate responses about $80 \%$ of the time to false negative questions.

There is one important implication of Choi's findings (1991) in regard to verification. Note that all children at the third stage used the agreement-disagreement dimension for true negative questions more often than for false negative questions. If children at this stage can be trained to verify statements, they should be able to verify true negatives better than false negatives. Since Korean-speaking children must verify before answering, they actually showed that true negatives are easier to verify than false negatives.

Young English-speaking children were able to respond successfully to negative questions but not to negative statements.

Akiyama and Guillory (1983) asked English-speaking children of ages 4 through 7 to verify statements and to answer corresponding questions using the within-subject design. Statements and questions were presented in a block format. The results are shown in the upper half of Table I. Generally, verifying statements was more difficult than answering corresponding questions $\left[\min F^{\prime}(1,52)=165.20 ; p<.001\right]$. The 4-year-olds found verifying negative statements extremely difficult and performed below a chance level (sign test, $z=2.25, p<.05$ ). On the average, 4-year-olds responded wrong to true negative statements and right to false statements on more than 7 out of 10 items. For example, a typical response to You aren't a baby was wrong. Five-year-olds also 
Table I. Error Rates for the Four Types of Statements and Four Types of Questions Among the Three Age Groups in English and Japanese

\begin{tabular}{|c|c|c|c|c|}
\hline Age & $\begin{array}{c}\text { True } \\
\text { affirmative }\end{array}$ & $\begin{array}{c}\text { False } \\
\text { affimative }\end{array}$ & $\begin{array}{c}\text { False } \\
\text { negative }\end{array}$ & $\begin{array}{c}\text { True } \\
\text { negative }\end{array}$ \\
\hline \multicolumn{5}{|c|}{ English statements (Akiyama \& Guillory, 1983) } \\
\hline 4 & .06 & .10 & .72 & .74 \\
\hline 5 & .02 & .04 & .54 & .56 \\
\hline $6-7$ & .01 & .04 & .10 & .28 \\
\hline \multicolumn{5}{|c|}{ English questions (Akiyama \& Guillory, 1983) } \\
\hline 4 & .03 & .07 & .02 & .07 \\
\hline 5 & .01 & .06 & .02 & .02 \\
\hline $6-7$ & .01 & .02 & .02 & .02 \\
\hline \multicolumn{5}{|c|}{ Japanese statements (Akiyama et al., 1982) } \\
\hline 4 & .03 & .17 & .63 & .26 \\
\hline 5 & .06 & .07 & .35 & .31 \\
\hline \multicolumn{5}{|c|}{ Japanese questions (Akiyama et al,, 1982) } \\
\hline 4 & .07 & .13 & .70 & .47 \\
\hline 5 & .04 & .04 & .49 & .55 \\
\hline
\end{tabular}

found verifying negatives somewhat difficult to do. Their performance was at chance level. It was not until age 6 that children became more able to verify statements.

On the other hand, answering yes-no questions was relatively easy (error rate .04) even for the youngest children. There was no difference between affirmative questions and negative questions. There was also no development of the answering system over the age range. These findings on questions were consistent with those of the oldest English-speaking children in Choi (1991).

Japanese-speaking children found answering negative questions more difficult than verifying negative statements.

Akiyama et al. (1982) examined the relative difficulty of verification and answering among the two age groups of Japanese-speaking children. As indicated in the lower half of Table $I$, answering questions was generally more difficult than verifying statements $[F(1,29)=15.27, p<$ $.01]$. This finding was opposite to that for English-speaking children in Akiyama and Guillory (1983).

Another finding was that verifying false-negative statements was 
more difficult than verifying true-negative statements for the 4-year-olds $[F(1,14)=9.31, p<.01]$. These data on 4-year-olds' in Japan were again opposite to those on the 6- to 7-year-olds in Akiyama and Guillory (1983). These results cast strong doubt on the idea that language processing is universal across languages and suggest the existence of two different strategies depending on the language.

In verification, English-speaking children and Korean-speaking children found true negatives more difficult than false negatives but the opposite was the case for Japanese-speaking children.

Akiyama (1984), Kim (1985), and Kim et al. (1990) have attempted to examine the effects of truth value and polarity only in statements. Akiyama (1984) used a task without pictures, Kim (1985) used a task with pictures, and Kim et al. (1990) used both. Since the results were similar across the tasks in Kim et al., the results on the two tasks were averaged in Table II. English-speaking children and Korean-speaking children found true negatives most difficult, followed by false negatives, and then by affirmatives. The three-way interaction between Truth Value $\times$ Polarity $\times$ Language was not significant (Kim et al., 1990). In contrast, Japanese-speaking children found false negatives most difficult, followed by true negatives, and then by affirmatives.

Compared to Table I, which indicates the verification performance

Table II. Error Rates for the Four Types of Statements in English-Speaking Children, Korean-Speaking Children, and Japanese-Speaking Children

\begin{tabular}{lccccc}
\hline & \multicolumn{5}{c}{ Statements } \\
\cline { 2 - 6 } \multicolumn{1}{c}{ Language } & Age & $\begin{array}{c}\text { True } \\
\text { affirmative }\end{array}$ & $\begin{array}{c}\text { False } \\
\text { affirmative }\end{array}$ & $\begin{array}{c}\text { False } \\
\text { negative }\end{array}$ & $\begin{array}{c}\text { True } \\
\text { negative }\end{array}$ \\
\hline $\begin{array}{l}\text { English } \\
\quad \text { (Akkiyama, 1984) }\end{array}$ & 3 & .08 & .12 & .41 & .52 \\
$\begin{array}{l}\text { English } \\
\quad \text { (Kim, 1985) }\end{array}$ & 3 & .03 & .08 & .21 & .54 \\
$\begin{array}{l}\text { English } \\
\quad \text { (Kim et al., 1990) }\end{array}$ & 4 & .06 & .02 & .15 & .48 \\
$\begin{array}{l}\text { Korean } \\
\quad \text { Kim, 1985) }\end{array}$ & 4 & .03 & .05 & .08 & .30 \\
$\begin{array}{l}\text { Korean } \\
\quad \text { Kim et al., 1990) }\end{array}$ & 4 & .03 & .04 & .24 & .73 \\
$\begin{array}{l}\text { Japanese } \\
\quad \text { (Akiyama, 1984) }\end{array}$ & 3 & .06 & .06 & .10 & .24 \\
\hline
\end{tabular}


by English-speaking children in Akiyama and Guillory (1983), the performance results shown in Table II are much better, with more contrast between false negatives and true negatives. This better performance can be explained by the fact that there was no question-answering task in this study. Without the question-answering task, errors due to confusion decreased. Across Tables I and II, there was a consistent tendency among English-speaking children and Japanese-speaking children. The older the children, the more contrast showed up between true and false negatives among English-speaking children, and the less contrast among Japanesespeaking children.

The finding that Korean-speaking children showed the same verification pattern as English-speaking children (Kim, 1985; Kim et al., 1990) poses a serious problem to Akiyama's (1984) proposal that children should show a similar pattern if their languages share similar typological characteristics described by Pope (1973). Typological characteristics may have nothing to do with how children process sentences. However, other linguistic characteristics of Korean used by young Korean-speaking children share some similarities with characteristics of Englich (Kim et al., 1990), which may help explain why Korean-speaking children and English-speaking children showed the same pattern. This aspect of the findings deserves further discussion.

The findings so far reviewed suggest that the only data that clearly support the Carpenter and Just (1975) model are those of the older English-speaking children and older Korean-speaking children. The younger English-speaking children who could not verify negative statements, and the younger Korean-speaking children and Japanese-speaking children of all ages seemed to verify statements differently from English-speaking adults. These findings call for a new conceptual framework.

Japanese-English bilingual children could answer English negative questions but could not answer Japanese negative questions correctly.

In an attempt to reevaluate a previous study (Akiyama, 1979), only those aspects that help clarify the relationship between verification and answering will now be discussed. The article examined the acquisition of the yes-no question answering system in three language groups, i.e., English monolinguals, Japanese monolinguals, and Japanese-English bilinguals who were generally more advanced in Japanese than in English. Their ages were 3 through 6 . Since the Japanese answering system is basically a verification system, the study can be interpreted as having compared the English monolinguals' answering system with the Japanese monolinguals' verification system and, at the same time, as having com- 
pared the bilinguals' English answering system with its verification system. Given that verification is more difficult than answering in English, two predictions can be made. One is that English monolinguals should find answering negative questions easier than do Japanese monolinguals. The other is that bilinguals should be better at answering English negative questions than at verifying Japanese negative statements. Bilinguals' performance on Japanese negative questions should be just like English monolinguals' performance on negative statements, since both groups have competing systems.

As shown in Table III, English-speaking children found negative questions slightly more difficult to answer than affirmative questions $(z=-2.00, p<.05$, the Wilcoxon test), whereas Japanese-speaking children found negative questions considerably more difficult to answer than affirmative questions. It is clear that English-speaking children found negative questions much easier to answer than Japanese-speaking children. The bilinguals' data were quite similar to those of the Englishspeaking children who showed extreme difficulty in verifying negative statements. Compare the verification part of Table I and the answering part for Japanese of Table II. While showing few errors on affirmatives, English-speaking children showed a high range of error rates (.54-.74) and Japanese-English bilinguals answering negative questions in Japanese showed a similar range (.57-.66). The effect was the same despite the different languages spoken by children.

Given the fact that these bilingual children had acquired the Japanese language and were generally more fluent in Japanese than in English, the findings strongly support the difficulty of verification over answering in English. The English answering system that does not require negative processing overrode the Japanese answering system among JapaneseEnglish bilingual children. Similarly, the English answering system ov-

Table III. Error Rates for Simple and Tag Questions in Monolingual and Bilingual Children

\begin{tabular}{|c|c|c|c|c|c|}
\hline \multirow[b]{3}{*}{ Group } & \multirow{3}{*}{$\begin{array}{c}\text { Language } \\
\text { used in } \\
\text { questions }\end{array}$} & \multicolumn{4}{|c|}{ Types of questions } \\
\hline & & \multicolumn{2}{|c|}{ Simple } & \multicolumn{2}{|c|}{ Tag } \\
\hline & & Affirmative & Negative & Affirmative & Negative \\
\hline \multirow[t]{2}{*}{ Monolingual } & English & .01 & .08 & .08 & .10 \\
\hline & Japanese & .00 & .19 & .09 & .22 \\
\hline \multirow[t]{2}{*}{ Bilingual } & English & .04 & .08 & .12 & .29 \\
\hline & Japanese & .07 & .66 & .15 & .57 \\
\hline
\end{tabular}


errode the verification system among English-speaking children. Children tended to use the simpler system in place of the more complex one when the two were in competition.

\section{DISCUSSION}

In this section I will describe how children verify statements and answer questions in different languages and address a few issues that call for clarification in future research. For this purpose, I use three information processing models. One is a verification model for English and Korean (e.g., Carpenter and Just, 1975; Clark and Chase, 1972; Kim, et al., 1990). The other is an answering model for English (Akiyama and Guillory, 1983). The third is a Japanese verification model (Akiyama, 1984) that can capture certain aspects of the verification pattern among Japanese-speaking children.

These three models have accounted for some findings reported in this article. First, the common core of these three models states that children comprehend true affirmative sentences such as You are a child in an identical manner regardless of the task (i.e., verification or answering) and language (i.e., English, Japanese, or any other language). The processing of affirmative true sentences is probably the typical case of language processing universal across languages.

Second, the answering model for English asserts that answering negative questions is inherently simpler than verifying negative questions in English. In this model, all English negative questions are affirmatively represented and, therefore, negatives need not be processed. Supporting this model, young English-speaking children find answering negative questions easier than verifying negative statements and young JapaneseEnglish bilinguals answer Japanese negative questions in the English way when they should use verification in answering their negative questions in Japanese. Furthermore, English-speaking children, French-speaking children, and Korean-speaking children in Choi (1991) showed the use of this answering model at the second stage. Thus, the implication of this model originally developed for English goes beyond English. Regardless of language-specific answering systems, children use this model initially for communication and ignore negative particles in negative questions. Ignoring negative particles in answering negative questions is probably another case of universal language processing among very young children.

Third, and most importantly, the verification models for English 
can account for the verification performance in both English-speaking children and Korean-speaking children and the verification model for Japanese can account for the verification performance in Japanese-speaking children. According to the models, English-speaking children find two mismatches in processing true negatives and one mismatch in processing false negatives. In contrast, Japanese-speaking children find no mismatch in processing true negatives and one mismatch in processing false negatives.

While these three models are reasonably successful in accounting for certain aspects of the findings, they do not explain four other important aspects. One is developmental in nature and concerns why young English-speaking children but not older children verify negative statements in a manner opposite to that specified by the model (e.g., You aren't a baby. Wrong!) and similarly, Japanese-English bilingual children answer Japanese negative questions in the English way (e.g., Are you not a baby? No!, when they are supposed to say right). This is a developmental question and the models proposed simply do not address this question.

The second aspect is that Japanese-speaking children find verification less difficult than answering. Since the two systems are basically identical in Japanese, they should be equally difficult.

The third aspect concerns the question of whether the model can assume that Japanese-speaking children derive negative knowledge representations in verification and answering. This is a representational question, which must be addressed with further evidence. These limitations are part of the inadequacy of information processing models in general. Tanenhaus, Carroll, and Bever (1976) have criticized these information processing models (e.g., that proposed by Carpenter and Just, 1975 ) on the grounds that they specify processes only after representations are formed and that the specification is ad hoc in nature.

The fourth aspect is that Korean-speaking children find true negative questions easier to answer than false negative questions (Choi, 1991), while they find true negative statements more difficult to verify than false negative statements (Kim et al., 1990). These four aspects are discussed in order.

\section{The Developmental Shift}

The developmental shift refers to the fact that young English-speaking children cannot verify negative sentences but older children can. One simple solution is to propose that English-speaking children initially have only one system - the answering system with a translation mechanism: 
yes is translated into right and no is translated into wrong. One system for verification and answering leads to no difference between them in affirmative sentences and a large difference in negative sentences.

The proposal that English children do not process negation at first is consistent with the observation that English-speaking children initially drop a negative when constructing a tag question (e.g., He'll catch cold, will he? from Brown \& Hanlon, 1970). Not only is this proposal valid for English-speaking children, it should work for children in other languages. When children cannot process a negation in verification, the only practical way of answering negative questions (e.g., Do you not want a cookie?) is to repeat a part of the sentence (e.g., want cookie or not cookie) without processing the whole sentence. Choi (1991) shows this tendency among French-speaking and Korean-speaking children and names it an elaborate response.

This type of strategy is far closer to the English answering system than to the Japanese answering system and should precede the Japanese answering system. As a matter of fact, a naturalistic observation of a Japanese-speaking child (Kokuritsu-Kokugo-Kenkyujo, 1982) shows a shift from a predominant use of nai (not) at ages 1;00 through 1;02 to a predominant use of un (yes) or uun (no) at age 1;04. In this case, nai is part of a previous sentence and yes or no is in response to a whole sentence. Thus, it is proposed that children, regardless of their languages, shift from a stage where they process parts of sentences without processing a negation to a stage where they process whole sentences with a negation.

\section{In Japanese, Verification is Easier than Answering}

The second finding to be accounted for is the better performance of the verification task than the answering task among Japanese-speaking children. Although the Japanese verification model can account for the relative difficulty of the four sentences within each of the tasks, it fails to predict better performance in verification than in answering. One easy solution to this problem is to adopt Clark and Clark's (1977) proposal that people answering yes-no questions initially affirm or deny the corresponding statements and then translate true or false into yes or no (Clark and Clark, 1977, pp. 100-102). Obviously, this strategy works for affirmatives but not for negatives in English. However, it works for both in Japanese.

In relation to the above assumption of verification being more basic than answering, presuppositions associated with negative questions should play a role. For the same reason that English negative questions are 
ambiguous (Stockwell, Schacter, and Partee, 1973), Japanese negative questions can be ambiguous (Kuno, 1973). Negative questions can be interpreted in at least two different ways depending on subtle intonation and context. For example, the two interpretations of the negative question Aren't you going? are as follows: One is to ask If it is true that you are not going? and the other is to ask for agreement, that is, I think you should agree with me that you should go. Japanese-speaking children may have had some difficulty in reaching the former-the verification interpretation.

The proposal that verification is more basic than answering is also supported by Noji's $(1974,1976)$ naturalistic observations. Many naturalistic observations, however, provide no information as to whether children's responses are correct. To be consistent with experimental reports, the examples that follow represent clear cases. The following is a discourse between a 22-month-old child and his father (Noji, 1976, p. 296):

Father: Pee?

Child: No.

Father: Right?

Child: Right.

Child: Right.

Child: Right (emphatically).

The child clearly understands that it is true that he does not want to go to the bathroom.

It took, however, four more months for the same child to be able to respond with yes-no answers such as un (yes) or $u$-un (no) (Noji, 1974, p. 76):

Mother: There wasn't (a sweet potato). You ate a lot.

Child: There wasn't. There wasn't.

Child: $\quad$ No (u-un). There was.

Although the child uses no, the context shows that he denies his own previous statement rather than answer a negative question.

A prototypical answering response to a negative question appeared even later at age 2;05 (Noji, 1974, p. 442):

Father: (Is a) cat not scary?

Child: Yes, (it is) not scary.

These examples show the early use of positive and negative morphemes to express agreement and disagreement with negative statements, thus supporting the primacy of verification over answering in Japanese- 
speaking children. The formulation based upon experimental findings is consistent with naturalistic data.

Another possible factor in accounting for verification primacy in Japanese and answering primacy in English is familiarity. One could argue that English-speaking children are exposed to yes-no questions far more frequently than to statements and that the reverse is the case for Japanese-speaking children. English-speaking children are indeed asked yes-no questions frequently (Steffensen, 1978). However, this is also the case with Japanese-speaking children, as indicated in the above examples. In addition, the analysis of a sample of 100 discourse units between a parent and a two-year-old boy (Noji, 1974, pp. 174-199) indicates 12 cases of yes-no question answering and no case of verification of statements. Thus, familiarity is confounded with the answering system in both languages. Familiarity alone cannot account for verification primacy in Japanese and answering primacy in English.

\section{Negative Knowledge Representations}

The form of representation assumed in the proposed models can be tested more directly. Note that the very process of verifying a false affirmative statement is a form of denial. In response to the statement You are a baby, for example, English-speaking children deny the statement and produce the knowledge representation $I$ am a child, while Japanese-speaking children produce the representation $I$ am not a baby.

To test this assumption, Akiyama (1985) designed a denial task in which two types of denial statements competed equally. In this task, children were asked to deny the statement $A$ ladybug is large. Their responses were then classified into affirmatives (e.g., A ladybug is small) and negatives (e.g., $A$ ladybug isn't large). According to the English verification model, children should produce affirmatives. According to the knowledge derivational model, children should produce negatives. The findings indicated that English-speaking children of 4 years of age used affirmatives $50 \%$ of the time and Japanese-speaking children of 4 years of age used affirmatives $23 \%$ of the time. In Kim et al. (1990), Korean-speaking children of 4 years of age used affirmatives $43 \%$ of the time. Korean-speaking children performed more like English-speaking children than Japanese-speaking children. These findings lend further support to the distinction made between the English verification models and the Japanese verification model.

Beyond these experimental findings, there is anecdotal evidence to support the greater availability of negative representations among Japanese-speaking children. Japanese-speaking children often deny a state- 
ment that includes negation. For example, two arguing Japanese-speaking children quite often say "You stupid are, " "I stupid not," "You stupid not not," "I stupid not not not," " and so forth. The Japanese translation of the last statement is Bokuwa (I) baka-ja (stupid) naku (not) naku (not) nai (not). Although this sentence sounds somewhat awkward, it is produced by some children and its sentence structure is judged as being grammatically correct by linguists (Saigusa, 1978). In a similar situation, in contrast, English-speaking children would say: "You are stupid." "No, I'm not," 'Yes, you are," "No, I'm not," 'Yes," "No," 'Yes," and so forth. This contrast seems to indicate a greater availability of negative representations among Japanese-speaking children.

A more general style characterization might be suggested to be compatible with all the evidence discussed above. There is a favored use of affirmative sentences in English (e.g., Strunk and White, 1972) and in Korean (Watanabe and Suzuki, 1981), and of negative sentences in Japanese (e.g., Christopher, 1983, p. 43; Ikegami, 1978). Negative sentences are avoided in English because of the ambiguity they entail. Precisely for the same reason, negative sentences are encouraged in Japanese. Clear-cut examples are found in asking questions. Suppose a Japanese speaker wants to ask a partner whether the partner goes to a certain place. The speaker is most likely to say "Iki (go)-masen (not)-ka (a question marker)" (the literal translation of this sentence is Are you not going?). In the same situation, the English speaker is most likely to say "Are you going?"

Another example may be found in a common saying. For example, Remember the Alamo in English is best translated into Japanese as Don't forget the Alamo. In child language, Clancy (1985) and McNeill and McNeill (1968) report a rich set of single-word negative expressions at the age of 2 . These include rejection, refusal, nonexistence, and denial. Clancy also remarks that the negation nai is a very frequent and salient marker of negation in Japanese (p. 394).

\section{Verification and Answering Among Korean-Speaking Children}

Since answering requires verification in Korean, performance in answering and performance in verification should match. However, there is a discrepancy. Verification studies (Kim, 1985; Kim et al., 1990) show that older children (ages 4-6) find true negatives more difficult to verify than false negatives. In contrast, answering studies (Choi, 1991) show that younger children (ages 2-3) can answer true negatives correctly $80 \%$ of the time but they produce elaborate responses to false negative questions. If these responses were coded according to the criterion of the 
use of morphemes, true negatives would result in $80 \%$ correct response and false negatives would result in fewer than $20 \%$ correct responses.

Two possibilities can be considered to resolve this unsettling issue. One is related to age in that there might be a developmental shift at around the earlier part of the age of 3 . The other is related to the task in that the answering and verification tasks demand different psychological processes.

\section{CONCLUSION}

The present article has attempted to show which processes are universal across languages and which are specific to a language in children's performance of verification and answering. Universal processes are: (a) All children regardless of their languages begin by using the English system and then later learn the Japanese or Korean system in answering negative questions and (b) all children verify affirmative statements and answer affirmative questions similarly. Specific processes are: (a) Verifying negative statements is more difficult than answering negative questions for English-speaking children but less difficult for Japanese-speaking children and (b) verifying true negatives is more difficult than verifying false negatives for English-speaking children and Korean-speaking children but less difficult for Japanese-speaking children.

The three information processing models proposed are successful in accounting for these findings. However, the three models alone cannot explain why English-speaking children in the studies totally failed to verify negative statements and why Japanese-speaking children found answering negative questions more difficult than verifying negative statements. To broaden the basis of these models, an attempt has been made to relate these models to three general language processing principles. They are: (a) Younger children use a simpler system in place of a more complex one if the two systems serve similar functions, (b) negative questions are generally more ambiguous than negative statements but the effects of ambiguity on comprehension are more pronounced in Japanese than in English, and (c) negative representations are more available and more preferred stylistically in Japanese than in English and Korean. Despite these attempts, one issue has emerged as unsettling. Younger Korean-speaking children find true negatives easier to answer than false negatives but find true negatives more difficult to verify than false negatives. 


\section{REFERENCES}

Akiyama, M. M. (1979). Yes-no answering systems in young children. Cognitive Psychology, 11, 485-504.

Akiyama, M. M. (1984). Are language acquisition strategies universal? Developmental Psychology, 20, 219-228.

Akiyama, M. M. (1985). Denials in young children from a cross-linguistic perspective. Child Development, 56, 95-102.

Akiyama, M. M., Brewer, B. F., \& Shoben, E. J. (1979). The yes-no question answering system and statement verification. Joumal of Verbal Leaming and Verbal Behavior, $18,365-380$.

Akiyama, M. M., \& Guillory, A. W. (1983). The ontogeny of the verification system. Journal of Verbal Learning and Verbal Behavior, 22, 333-340.

Akiyama, M., Takei, S., \& Saito, K. (1982). Statement verification and question answering. Japanese Journal of Educational Psychology, 30, 215-222.

Brown, R. (1973). A first language. Cambridge, MA: Harvard University Press.

Brown, R., \& Hanlon, C. (1970). Derivational complexity and order of acquisition in child speech. In J. R. Hayes (ed.), Cognition and the development of language. New York: John Wiley and Sons.

Carpenter, P., \& Just, M. A. (1975). Sentence comprehension: A psycholinguistic model of sentence verification. Psychological Review, 82, 45-73.

Choi, S. (1991). Children's answer to yes-no questions: A developmental study in English, French, and Korean. Developmental Psychology, 27, 407-420.

Christopher, R. C. (1983). The Japanese mind. New York: Fawcett Columbine.

Clancy, P. M. (1985). The acquisition of Japanese. In D. I. Slobin (Ed.), The crosslinguistic study of language acquisition: Vol. 1. The data. Hillsdale, NJ: Erlbaum.

Clark, H. H. (1973). The language-as-fixed effect fallacy: A critique of language statistics in psychological research. Joumal of Verbal Learning and Verbal Behavior, 12, 335-359.

Clark, H. H., \& Chase, W. G. (1972). On the process of comparing sentences against pictures. Cognitive Psychology, 3, 472-517.

Clark, H. H., \& Clark, E. V. (1977). Psychology and language. New York: Harcourt Brace Jovanovich.

Ikegami, Y. (1978). Imino sekai [The world of]. Tokyo: Nippon Hoso Shuppan Kyokai. Kim, K. J. (1985). Development of the concept of truth functional negation. Developmental Psychology, 21, 426-472.

Kim, J. L., Shatz, M., \& Akiyama, M. M. (1990). Developmental Psychology, 26, 821-829.

Klima, E. S., \& Bellugi, U. (1973). Syntactic regularities in the speech of children. In C. A. Ferguson \& D. I. Slobin (Eds.), Studies of child language development (pp. 333-355). New York: Holt, Rinehart \& Winston.

Kokuritsu-Kokugo-Kenkyujo (1982). Yoji-no kotoba shiryo [Child language data] Vol. 3. Tokyo: Shuei-shuppan.

Kuno, S. (1973). The structure of the Japanese language. Cambridge, MA: The MIT Press.

McNeill, D., \& McNeill, N. B. (1968). What does a child mean when he says "no?" In C. A. Ferguson \& D. I. Slobin (Eds.), Studies in child language development (pp. 619-627). New York: Holt, Rinehart \& Winston.

Noji, J. (1974). Yojiki no gengo seikatsu no jittai [Child language record] Vol. 3. Hiroshima: Bunka Hyoronsha. 
Noji, J. (1976). Yojikino gengo seikatsu no jittai [Child language record] Vol. 2. Hiroshima: Bunka Hyoronsha.

Okubo, A. (1967). The development of child language [Yoji gengo-no hattatsu]. Tokyo: Tokyodo Shuppan.

Pope, E. (1973). Question answering systems. Paper presented at the 9th regional meeting of the Chicago Linguistic Society, Chicago.

Saigusa, M. (1978). Nihon bunpo [Japanese grammar]. Tokyo: Iwanami Shoten.

Steffensen, M. S. (1978). Satisfying inquisitive adults: Some simple methods of answering yes/no questions. Joumal of Child Language, 5, 221-236.

Stockwell, R., Schacter, P., \& Partee, B. (1973). The major syntactic structure of English. New York: Holt, Rinehart and Winston.

Strunk, W., \& White, E. B. (1972). The elements of style. New York: Macmillan.

Tanenhous, M. K., Carroll, J. M., \& Bever, T. G. (1976). Sentence picture verification models as theories of sentence comprehension: A critique of Carpenter and Just. Psychological Review, 83, 310-317.

Watanabe, K., \& Suzuki, T. (1981). Chosengo-no susume [Invitation to Korean]. Tokyo: Kodansha. 